\title{
In Vitro Translation of the Intermediate Filament Proteins Desmin and Vimentin
}

\author{
CLARE M. O’CONNOR, $†$ DAVID J. ASAI, $\ddagger$ CONSTANTIN N. FLYTZANIS, AND ELIAS LAZARIDES* \\ Division of Biology, California Institute of Technology, Pasadena, California 91125
}

Received 9 December 1980/Accepted 29 January 1981

\begin{abstract}
Polyadenylated ribonucleic acid (RNA) was isolated from chicken skeletal and smooth muscle and translated in a cell-free rabbit reticulocycle system. Both types of muscle tissue contain messenger RNAs that code for the intermediate filament proteins desmin and vimentin, and the relative concentrations of the two translation products reflect the prevalence of the two proteins in vivo. Desmin synthesis represents a greater proportion of the total protein synthesis from smooth muscle RNA than from skeletal muscle RNA, whereas the converse is true of vimentin synthesis. Fractionation of the RNA on formamide-containing sucrose gradients before translation indicates that the desmin messenger RNA is larger than the vimentin messenger RNA and contains an extensive noncoding segment. The desmin and vimentin messages code predominantly for the nonphosphorylated forms of desmin and vimentin. However, the ratio of phosphorylated to unphosphorylated forms of the proteins could be increased by adding cyclic adenosine monophosphate-dependent kinase activity to the translation mixtures. These results suggest that desmin and vimentin are each synthesized from a single messenger RNA species and that posttranslational phosphorylation generates the additional isoelectric variants of each which are observed in vivo.
\end{abstract}

Electron microscopic studies have demonstrated that filaments with a diameter of $10 \mathrm{~nm}$ are ubiquitously distributed in eucaryotic cells (reviewed in reference 9). Since this diameter is intermediate to that of cytoplasmic actin $(6 \mathrm{~nm})$ and myosin $(15 \mathrm{~nm})$ filaments, the 10-nm filaments were originally termed intermediate filaments. However, despite their morphological similarity, recent evidence from many immunological and biochemical studies has established that there are several classes of intermediate filaments which can be distinguished by their subunit protein(s). Five major filament classes have been described: neurofilaments, glial filaments, keratin filaments, smooth muscle desmin filaments, and the vimentin filaments found in cells of mesenchymal origin. The distributions of the different intermediate filament classes are not exclusive; more than one class of filaments is frequently observed in the cytoplasm of an individual cell.

Both desmin and vimentin are present in muscle cells and in fibroblastic cells (3). Immunofluorescent localization studies have shown that desmin and vimentin are associated with fila-

† Present address: Department of Chemistry, University of California at Los Angeles, Los Angeles, CA 90024.

$\ddagger$ Present address: Department of Biology, University of California at Santa Barbara, Santa Barbara, CA 93106. mentous networks in the cytoplasm of fibroblasts, myoblasts, and young myotubes, whereas in mature muscle cells, both proteins are concentrated at the Z-line $(4,5)$. In addition, since neither protein is extracted from the cells by extraction with nonionic detergents, desmin and vimentin have been identified as cytoskeletal proteins $(3,13)$. When muscle cell cytoskeletal proteins are electrophoresed on two-dimensional gels, both desmin and vimentin show microheterogeneity $(8,13)$. Desmin appears as a set of isoelectric variants with a molecular weight of 50,000 , and vimentin appears as a series of variants with a molecular weight of 52,000 . Two major isoelectric variants of desmin, $\alpha$-desmin and $\beta$-desmin, and several minor variants are resolved. Previous experiments have demonstrated that $\alpha$-desmin and the minor variants are phosphorylated proteins but that $\beta$-desmin is not phosphorylated (13).

Several phosphorylated variants of vimentin are also present in muscle cells, but usually the unphosphorylated variant predominates (13). With both desmin and vimentin, it remains unclear whether a single gene product is phosphorylated post-translationally to give rise to all of the variants or whether several different gene products are synthesized.

Although the data concerning desmin and vi- 
mentin suggest that they are distinct protein species, some of their physical and chemical properties are remarkably similar (16). Comparative peptide mapping has also suggested some sequence homology between the proteins $(3,16)$. These hypothetical regions of sequence homology probably do not include the major antigenic determinants, however, since antisera prepared against desmin and vimentin do not generally cross-react (5).

In the experiments described in this paper, we have attempted to resolve the questions about desmin and vimentin heterogeneity posed above by in vitro translation of skeletal and smooth muscle messenger ribonucleic acid (mRNA) in a cell-free reticulocyte lysate. In all cases, both desmin synthesis and vimentin synthesis are detected. Separation of the desmin and vimentin mRNA's by their size provides definitive proof for the separate identities of desmin and vimentin, ruling out post-translational conversion of vimentin to desmin. The principal translation product of desmin mRNA is $\beta$-desmin. The ratio of $\alpha$ - to $\beta$-desmin synthesized in the cell-free system can be increased by supplementing the lysate with the catalytic subunit of the bovine heart cyclic adenosine monophosphate (cAMP)dependent protein kinase, suggesting that $\alpha$-des$\mathrm{min}$ is derived from $\beta$-desmin by post-translational phosphorylation.

\section{MATERIALS AND METHODS}

RNA preparation. Gizzard and thigh muscles were dissected from 1- to 7-day old chicks and frozen in liquid nitrogen. The frozen tissue was pulverized to a fine powder in liquid nitrogen, using a Waring blender with a stainless-steel cup. Total RNA was extracted from the frozen, pulverized tissue by using guanidine thiocyanate, as described by Chirgwin et al. (1). The RNA was resuspended in the guanidine- $\mathrm{HCl}$ solution and precipitated twice. The pelleted RNA was resuspended in distilled water and precipitated overnight at $-20^{\circ} \mathrm{C}$ with 0.1 volume of $2 \mathrm{M}$ potassium acetate, $\mathrm{pH}$ 5 , and 2.5 volumes of cold $\left(-20^{\circ} \mathrm{C}\right)$ ethanol before oligodeoxythymidylate [oligo(dT)]-cellulose fractionation. After pelleting at $16,000 \times g$ for $10 \mathrm{~min}$, the RNA was resuspended in $10 \mathrm{mM}$ tris(hydroxymethyl)aminomethane (Tris), pH 7.4-1 $\mathrm{mM}$ ethylenediaminetetraacetate (EDTA), heated at $60^{\circ} \mathrm{C}$ for $5 \mathrm{~min}$, $60^{\circ} \mathrm{C}$ for $5 \mathrm{~min}$, and chilled rapidly on ice. An equal volume of the same buffer with $1 \mathrm{M} \mathrm{NaCl}$ was added to the sample, which was then passed through an oligo(dT)-cellulose column (0.2 g) four times. After washing with the same buffer, the RNA was eluted with 3 column volumes of $10 \mathrm{mM}$ Tris ( $\mathrm{pH} 7.4)-1 \mathrm{mM}$ EDTA and precipitated with potassium acetate and ethanol as above.

Fractionation of RNA. The polyadenylic acid [poly(A)]-containing RNA was dissolved in $3 \mathrm{mM}$ Tris (pH 7.5)-3 mM EDTA-70\% formamide, heated at $60^{\circ} \mathrm{C}$ for $5 \mathrm{~min}$, and centrifuged over a 5 to $20 \%$ linear sucrose gradient in $3 \mathrm{mM}$ Tris (pH 7.5)-3 mM EDTA$70 \%$ formamide for $38 \mathrm{~h}$ at $39,000 \mathrm{rpm}$ and $20^{\circ} \mathrm{C}$ in a Beckman SW41 rotor (17). The gradient was fractionated by collecting drops from the bottom of the tube, and the RNA in each fraction was precipitated with potassium acetate and ethanol as above. After centrifugation, the pellets were extracted twice with $70 \%$ ethanol and the RNA was dissolved in water. RNA from every second fraction of the gradient was used for translation in the cell-free lysate.

Cell-free translation of RNA. The RNA which bound to oligo(dT)-cellulose was pelleted by centrifugation at $16,000 \times g$ for $10 \mathrm{~min}$ and resuspended in water at a concentration of $1 \mathrm{mg} / \mathrm{ml}$. The resuspended RNA was heated at $60^{\circ} \mathrm{C}$ for $5 \mathrm{~min}$ and rapidly chilled on ice. The RNA was translated in a rabbit reticulocyte lysate according to the manufacturer's specifications.

The final concentrations of components in the translation mixture were $80 \mathrm{mM}$ potassium acetate, $0.65 \mathrm{mM}$ magnesium acetate and $50 \%$ (vol/vol) reticulocyte lysate. Each 25- $\mu$ - translation mixture contained 0.5 to $1.0 \mu \mathrm{g}$ of muscle RNA and $50 \mu \mathrm{Ci}$ of $\left[{ }^{35} \mathrm{~S}\right]$ methionine $(1,036 \mathrm{Ci} / \mathrm{mmol})$. The standard incubation was at $37^{\circ} \mathrm{C}$ for $60 \mathrm{~min}$. Incorporation of methionine into protein was linear over this period. After various times of incubation, $1 \mu$ l of the translation mixture was removed, and the protein was precipitated as described by Devlin and Emerson (2). One microliter of sample mixture contained 50,000 to 80,000 counts which were precipitable with $10 \%$ (vol/vol) trichloroacetic acid. The reaction was terminated by adding 3 volumes of $8 \mathrm{M}$ urea-2\% Nonidet P-40-0.5\% 2-mercaptoethanol to the translation mixture and immediately freezing the sample on dry ice.

For pulse-chase experiments, translation was performed as above for $10 \mathrm{~min}$. After $10 \mathrm{~min}$ of incubation, $1 \mu \mathrm{l}$ of $100 \mathrm{mM}$ nonradioactive methionine was added to the lysate. The incorporation of radioactive methionine into trichloroacetic acid-precipitable material ceased immediately. One microliter of either cAMP. dependent kinase $(11,000 \mathrm{U} / \mathrm{ml})$ or buffer was then added to the reaction mixture. Incubations were allowed to proceed for various lengths of time.

Two-dimensional gel electrophoresis and fluorography. Translation samples, containing $1.0 \times 10^{6}$ to $1.6 \times 10^{6}$ trichloroacetic acid-precipitable counts, were mixed with $10 \mu \mathrm{g}$ of chicken skeletal muscle cytoskeletal proteins in the same urea sample buffer. The cytoskeletal marker proteins were prepared by detergent extraction of cultured primary chicken muscle cells as described previously (13). Samples were electrophoresed on two-dimensional isoelectric focusing-sodium dodecyl sulfate-polyacrylamide slab gels as described previously (10). Gels were prepared for fluorography by impregnation with Enhance solution for $1 \mathrm{~h}$. After rinsing with deionized water for an additional hour, the gels were dried under vacuum. Autoradiograms were exposed for 2 to 3 days at $-70^{\circ} \mathrm{C}$ before development as described previously (13).

Guanidine thiocyanate was purchased from Tridom Chemicals, Hauppage, N. Y. Oligo(dT)-cellulose (type III) was purchased from Collaborative Research, Inc., Waltham, Mass. The rabbit reticulocyte cell-free translation kit and Enhance solution were obtained 
from New England Nuclear Corp., Boston, Mass. The catalytic subunit of bovine heart cAMP-dependent protein kinase (P2645) was purchased from Sigma Chemical Co., St. Louis, Mo. Ampholines for isoelectric focusing were purchased from LKB Instruments Inc., Rockville, Md. Kodak XR-5 film was used for autoradiography.

\section{RESULTS}

Translation of skeletal and smooth muscle RNA in vitro. mRNA was isolated from total RNA of smooth (gizzard) and skeletal (thigh) muscle tissues by oligo(dT)-cellulose chromatography. The RNA prepared by this procedure efficiently directs the synthesis of fulllength proteins in the cell-free system derived from rabbit reticulocytes. Control experiments indicated that this poly $(\mathrm{A})^{+}$-enriched fraction of RNA contained most of the messenger activity of the muscle RNA. Figure 1 shows the in vitro translation products of the two RNA populations. Actin is the principal product of both the skeletal and the smooth muscle RNAs. It has been reported previously that $\alpha$-actin is the principal translation product in skeletal muscle (7), whereas $\gamma$-actin is the principal isoelectric variant synthesized in smooth muscle (14). The present results confirm these observations, although it is clear from the gels shown in Fig. 1 (which has been overexposed to emphasize the desmin and vimentin) that other actin variants are also synthesized. It is not clear whether this is due to the presence of nonmuscle cells in the muscle tissue or to incorrect translation in the cell-free system. It is also possible to distinguish translation products which comigrate with $\delta$ and $\epsilon$-actin, the non-acetylated precursor of $\beta$ and $\gamma$-actin $(7,14)$.

The principal desmin synthesized from either skeletal or smooth muscle RNA is $\beta$-desmin. Smaller amounts of $\alpha$-desmin are also synthesized. The ratio of $\beta$-desmin to $\alpha$-desmin synthesis in the cell-free system, determined by densitometry of autoradiograms (exposed for short periods of time to ensure a linear film response) averages $10: 1$. This value is significantly greater than the ratio of the $\beta$ - to $\alpha$-desmin polypeptides observed in intact tissues, which is usually about 1:1 $(4,8)$. The ratio of the two isoelectric variants is similar in translations of smooth and skeletal muscle RNA. Comparison of the translation products from skeletal and smooth muscle RNA, however, shows that synthesis of desmin in vitro from smooth muscle mRNA is much higher than from skeletal muscle mRNA.

Vimentin is a readily detectable product of translation of skeletal muscle mRNA, where its concentration approaches that of desmin. However, in the translation of smooth muscle

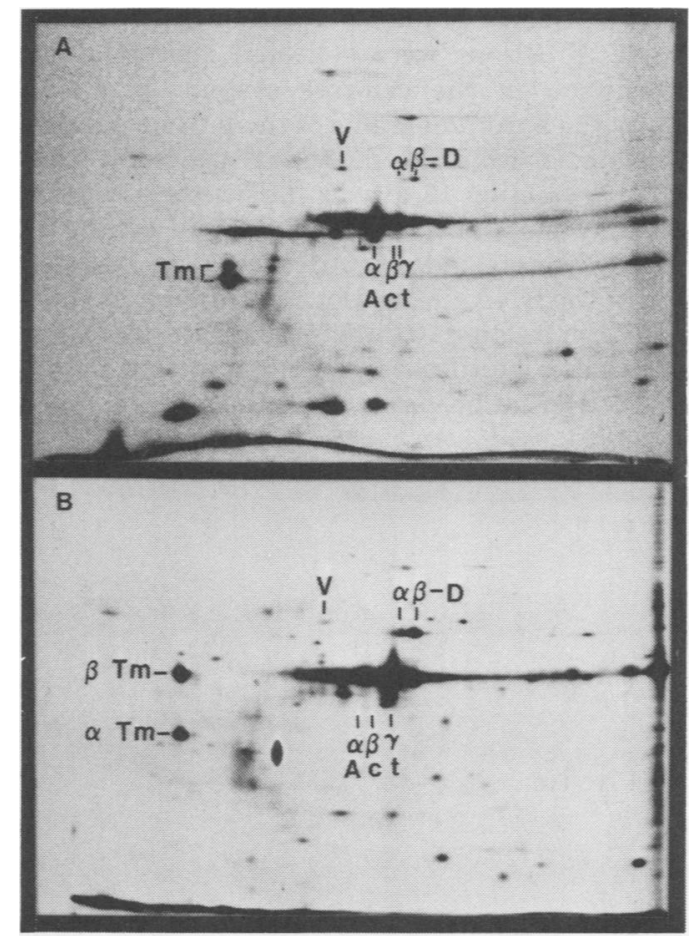

Fig. 1. Translation of skeletal and smooth muscle $R N A$ in vitro. The $\left[{ }^{35} S\right]$ methionine-labeled translation products of skeletal $(A)$ and smooth (B) muscle $\operatorname{poly}(A)^{+}$RNA were electrophoresed on two-dimensional isoelectric focusing-sodium dodecyl sulfate gels. The acid end of the gel is on the left. Sodium dodecyl sulfate electrophoresis is from top to bottom. The positions of actin (Act), desmin (D), vimentin (V), and tropomyosin (Tm) are indicated.

mRNA, vimentin synthesis is always far less than that of desmin. Thus, the concentrations of the two intermediate filament proteins synthesized in vitro reflects the prevalence of the proteins in the intact tissues $(4,5)$. The major isoelectric variant of vimentin translated in vitro from both smooth and skeletal muscle RNAs corresponds to the unphosphorylated form of vimentin (13).

A further distinction between the RNA populations from smooth and skeletal muscle is apparent in the kinds of tropomyosins synthesized in vitro. Skeletal and smooth muscle RNAs code for tropomyosin species which are electrophoretically distinct (8). The tropomyosins synthesized from mRNA in vitro comigrate with the tropomyosin species present in the tissues of origin.

Fractionation of desmin and vimentin mRNA's. Skeletal muscle poly(A) ${ }^{+}$RNA was fractionated by ultracentrifugation through a 5 
to $20 \%$ sucrose gradient containing $70 \%$ formamide. Fractions were collected and used for translation in the cell-free system. Figure 2 shows the autoradiograms of the protein species synthesized from the individual fractions. The RNA in fraction 13 directs the synthesis of the tropomyosins most efficiently. Actin synthesis in vitro is detected primarily from the translation of the RNAs in fractions 13 through 15. The mRNA in fraction 15 directs the greatest amount of vimentin synthesis. Desmin is found primarily in the translation products of the RNA in frac- tions 17 through 19. Further studies have demonstrated that the actual peak of desmin mRNA activity occurs in fraction 18 . The modal size of the RNA in this latter fraction has been determined as $2.1 \pm 0.2$ kilobases by electrophoresis on a methyl mercury-containing agarose gel (data not shown). Two-dimensional gel electrophoresis of the proteins synthesized from the different RNA fractions demonstrates that separate mRNA's for the $\alpha$ - and $\beta$-isoelectric variants of desmin are not separated on the formamide gradient.
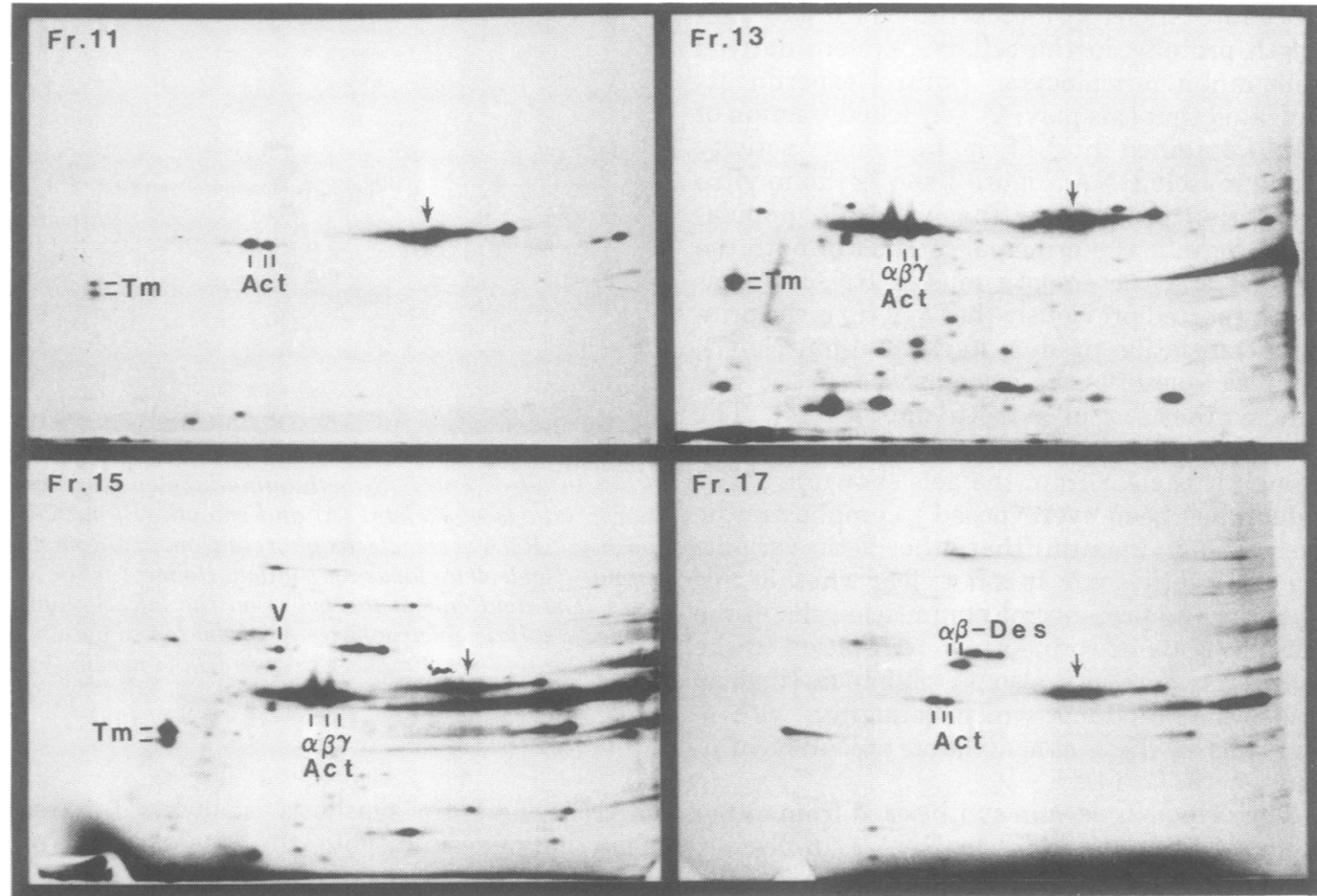

Fr.17
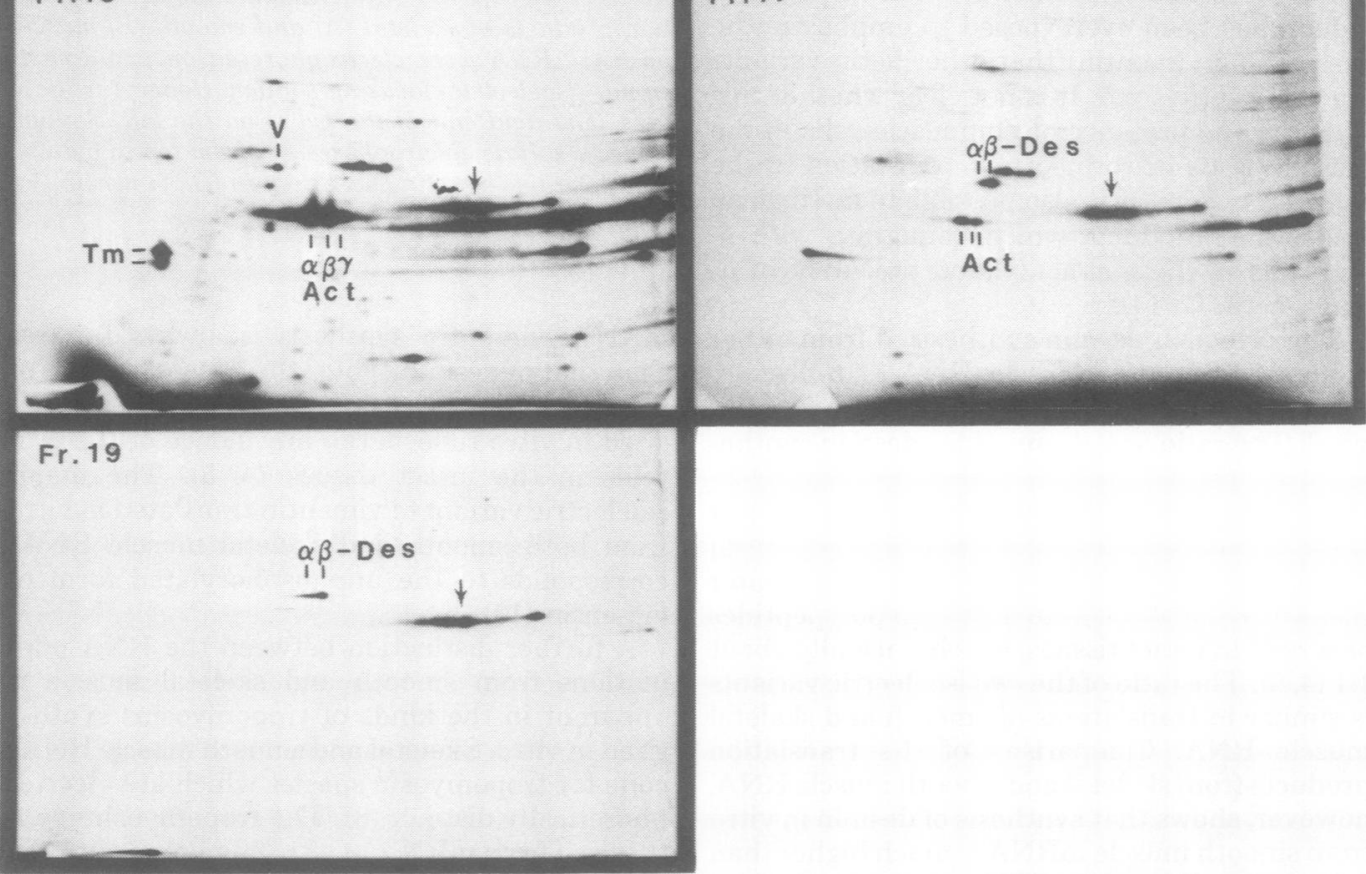

Fig. 2. Fractionation of skeletal muscle RNA. Skeletal muscle poly $(A)^{+} R N A$ was fractionated on a sucrose gradient containing $70 \%$ formamide. The translation products of the RNA in fractions $11,13,15,17$, and 19 were separated on two-dimensional isoelectric focusing-sodium dodecyl sulfate gels. The positions of desmin (Des), actin (Act), tropomyosin (Tm), and vimentin (V) are indicated. Arrows point to a translation product of the reticulocyte lysate $R N A$. 
Generation of $\alpha$-desmin from $\beta$-desmin by post-translational phosphorylation. Our previous experiments have demonstrated that $\alpha$-desmin is a phosphorylated protein, but that $\beta$-desmin is not (13). These experiments also demonstrated that the cAMP-dependent kinases phosphorylated desmin in vitro at the same sites that were phosphorylated in vivo (13a). However, it was not possible to determine in these experiments whether there was a single desmin polypeptide synthesized in muscle cells which acted as a substrate for several different phosphorylation reactions, or whether several different desmin polypeptides were synthesized in cells, only some of which became phosphorylated. These possibilities were tested directly by supplementing the reaction mixture containing lysate and smooth muscle mRNA with exogenous cAMP-dependent kinase catalytic subunit immediately after the addition of an excess of nonradioactive methionine. The kinase was not added during the labeling period because of its reported effect on the translation process (12). By 10 min after the addition of kinase, the ratio of $\alpha$ - to $\beta$-desmin detected on the autoradiograms was increased severalfold over the control value (Fig. 3). In addition, several minor, more acidic variants of desmin were also apparent. These minor variants are often observed in vivo $(4,13)$, but they are not observed normally among the translation products of muscle mRNA. The effect of the exogenous kinases was a transitory one. By 30 min after the addition of kinase, the ratio of $\alpha$ - to $\beta$-desmin was similar in both control and kinase-treated lysates. This may be due to the action of the endogenous lysate protein phosphatases.
Similar results were observed with vimentin (when fluorograms were exposed for longer periods of time). The addition of kinase activity to the lysate augmented the proportion of the more acidic vimentin variants relative to the major variant. We have previously observed that these acidic variants are phosphoproteins, whereas the major variant is not (13). Again, the effect of the exogenous kinase was a transitory one, disappearing within $30 \mathrm{~min}$ of kinase addition.

\section{DISCUSSION}

The cell-free translation experiments reported here have been instructive in defining intermediate filament protein heterogeneity. It is clear from the data that desmin and vimentin are the translation products of discrete mRNA molecules which can be physically separated. Desmin cannot, therefore, be regarded as a proteolytic fragment of vimentin. Instead, the marked similarities in the physical and chemical properties of desmin and vimentin and of their respective filaments may reflect the evolutionary derivation of the two proteins from a common ancestral protein (3). The relative concentrations of desmin and vimentin products found in the translation of smooth and skeletal muscle RNA are very similar to their relative concentrations observed in vivo. Assuming that desmin and vimentin are translated with comparable efficiency both in vitro and in vivo, this implies that the tissue concentrations of the two proteins are regulated by the concentrations of their respective mRNA's.

Using formamide-containing sucrose gradients (Fig. 2) or nondenaturing sodium dodecyl sulfate gel electrophoresis (C. O'Connor and D.

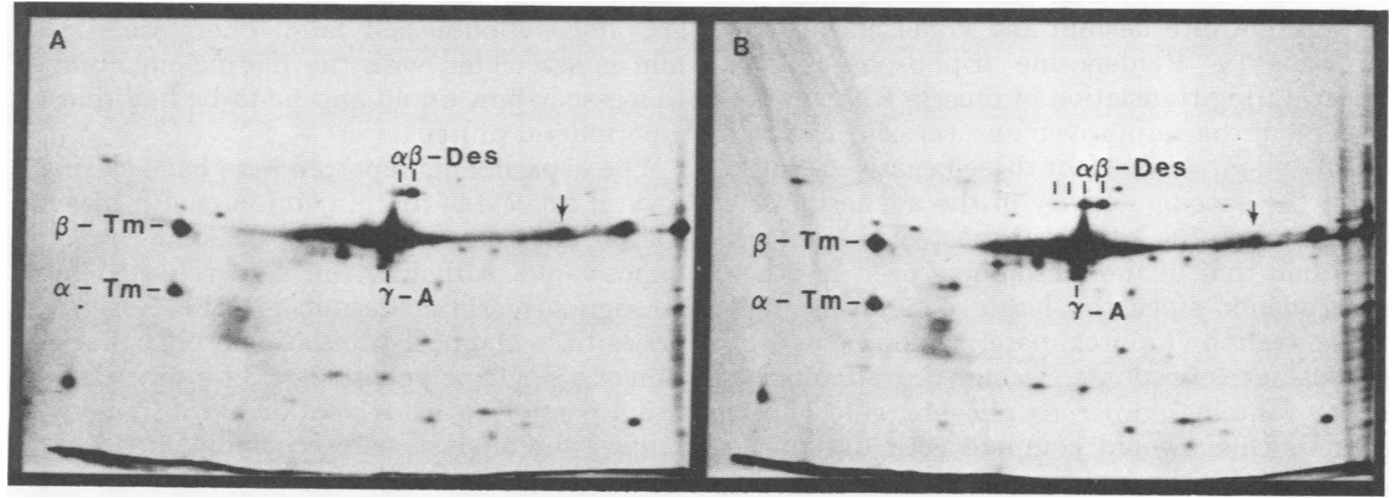

Fig. 3. Post-translational phosphorylation of $\beta$-desmin. Gizzard poly $(A)^{+}$RNA was translated for 10 min before the addition of an excess of nonradioactive methionine to the lysate. Samples were subsequently incubated for $10 \mathrm{~min}$ in the absence $(A)$ or presence $(B)$ of bovine heart muscle cAMP-dependent kinase activity. Translation products were separated by two-dimensional isoelectric focusing-sodium dodecyl sulfate gels. The positions of actin (Act), tropomyosin (Tm), desmin (Des), and a reticulocyte translation product (arrows) are indicated. 
Asai, unpublished data), we have not detected separate mRNA's for the different isoelectric variants of desmin. The desmin mRNA was surprisingly large for a protein with a molecular weight of 50,000 . We estimate that a noncoding stretch of 900 nucleotides is present in the message, since the total number of amino acids in desmin is approximately $400(6,15)$. All fractions containing this desmin mRNA activity directed the synthesis of predominantly $\beta$-desmin in vitro, although small amounts of $\alpha$-desmin were also synthesized. Since rabbit reticulocyte extracts are known to contain appreciable amounts of endogenous cAMP-dependent kinase activity (12), it was possible that $\alpha$-desmin was being derived from $\beta$-desmin by post-translational phosphorylation occurring in the lysate. This possibility was tested by adding kinase after the translation period and comparing the amounts of newly synthesized $\alpha$-desmin and $\beta$-desmin in the presence or absence of added kinase. Whereas the ratio of the $\alpha$-desmin to $\beta$-desmin translated in vitro in the absence of added kinase was consistently much lower than the molar ratio of the two variants in vivo, the $\alpha$-desmin to $\beta$-desmin ratio was dramatically increased by adding the catalytic subunit of the bovine heart cAMP-dependent protein kinase to the lysate during a chase period initiated by a large excess of nonradioactive methionine. Therefore, $\alpha$-desmin can be directly generated from $\beta$-desmin by post-translational phosphorylation. Together with the fractionation data, this suggests that the desmin isoelectric variants are derived from a single polypeptide by post-translational phosphorylation. This is in contrast to actin, whose three isoelectric variants are translated from different mRNA's (7).

We have attempted to observe phosphate incorporation into desmin and vimentin directly by using $\left[\gamma^{3}{ }^{32} \mathrm{P}\right]$ adenosine triphosphate as a tracer during translation of muscle RNA in the lysate, but this has proven unsuccessful. Several factors might account for these negative results. First, the specific activity of the adenosine triphosphate in the lysate is expected to be much less than that of the methionine used in other experiments, since the lysate contains a high concentration (approximately millimolar) of adenosine triphosphate. Second, the reticulocyte lysate contains numerous phosphate-accepting species which would compete with desmin as substrates for the endogenous kinases (13). We did, in fact, observe phosphate incorporation into a large number of reticulocyte proteins. These negative results do not detract, however, from the major conclusion. The results reported above demonstrate that $\alpha$-desmin (and the mi- nor acidic variants) can be derived from $\beta$-desmin by the action of cAMP-dependent protein kinases.

The ability of kinases to generate $\alpha$-desmin from $\beta$-desmin in the cell-free system also demonstrates that soluble desmin can be phosphorylated. Since only minute quantities of desmin are synthesized in vitro and since the reticulocyte lysates do not themselves contain detectable desmin, the concentration of desmin in the lysate should be well below the critical concentration for its polymerization (6). The efficient phosphorylation of soluble desmin which we have demonstrated here may have important functional implications. When detergent-resistant structures containing desmin are isolated from cells, they always contain nearly equal amounts of the $\alpha$-desmin and $\beta$-desmin variants $(3,13)$. It is therefore possible that desmin phosphorylation is required for one of the early steps in its assembly into stable cytoskeletal structures.

Immunofluorescent localization studies with specific desmin antibodies have shown that desmin is frequently associated with cytoplasmic structures where actin filaments insert into membranes $(9,11)$. On the basis of these data, it has been proposed that desmin might function as a link between actin filaments and the plasma membrane by virtue of its hydrophobic properties. The results reported here, however, suggest that desmin biosynthesis is unlike that of many integral membrane proteins in that mature-sized desmin is translated without a hydrophobic leader sequence (18). This mature-sized desmin can be specifically precipitated from the translation mixtures with desmin antibodies; no larger proteins (including vimentin) are specifically precipitated with the antibodies (Asai and O'Connor, unpublished data). Therefore, if desmin is associated with the plasma membrane, this association would appear to be like that of a peripheral protein.

The experiments reported here have clarified several aspects of desmin and vimentin biosynthesis, but some important questions remain unanswered. Although the data reported here strongly suggest that desmin microheterogeneity arises from the post-translational phosphorylation of a single polypeptide, strict interpretation is not possible. Final resolution of this point will require the analysis with recombinant deoxyribonucleic acid probes of the RNA species derived from active desmin genes. Such probes may also be useful for investigating the fine structure of the desmin and vimentin genes. Our results have clearly demonstrated that desmin and vimentin are distinct gene products and that 
desmin phosphorylation can be closely linked to its biosynthesis. It will be interesting to examine the effect of desmin phosphorylation on filament assembly in a defined cell-free system where the level of phosphorylation can be manipulated experimentally.

\section{ACKNOWLEDGMENTS}

This work was supported by grants from the Public Health Service (GM-06965, National Institute of General Medical Science), National Science Foundation, and the Muscular Dystrophy Association of America and by a Biomedical Research Support Grant to the Division of Biology. C.M.O. and D.J.A. were supported by postdoctoral fellowships from the Muscular Dystrophy Association of America; C.N.F. was supported by a postdoctoral fellowship from the German Cancer Research Center, Heidelberg, Germany. Elias Lazarides is a recipient of a Public Health Service Research Career Development Award from the National Institutes of Health.

\section{LTERATURE CITED}

1. Chirgwin, J. M., A. E. Przybyla, R. J. MacDonald, and W. J. Rutter. 1979. Isolation of biologically active ribonucleic acid from sources enriched in ribonuclease. Biochemistry 18:5294-5299.

2. Devlin, R. B., and C. P. Emerson, Jr. 1979. Coordinate accumulation of contractile protein mRNAs during myoblast differentiation. Dev. Biol. 69:202-216.

3. Gard, D. L., P. B. Bell, and E. Lazarides. 1979. Coexistence of desmin and the fibroblastic intermediate filament subunit in muscle and nonmuscle cells: identification and comparative peptide analysis. Proc. Natl. Acad. Sci. U.S.A. 76:3894-3898.

4. Gard, D. L., and E. Lazarides. 1980. The synthesis and distribution of desmin and vimentin during myogenesis in vitro. Cell 19:263-275.

5. Granger, B. L., and E. Lazarides. 1979. Desmin and vimentin coexist at the periphery of the myofibril $\mathrm{Z}$ disc. Cell 18:1053-1063.

6. Huiatt, T. W., R. M. Robson, N. Arakawa, and M. H. Stromer. 1980. Desmin from avian smooth muscle. Purification and partial characterization. J. Biol. Chem. 255:6981-6989.
7. Hunter, T., and J. I. Garrels. 1977. Characterization of the mRNAs for $\alpha$ - $\beta$ - and $\gamma$-actin. Cell 12:767-781.

8. Izant, J. G., and E. Lazarides. 1974. Invariance and heterogeneity in the major structural and regulatory proteins of chick muscle cells revealed by two-dimensional gel electrophoresis. Proc. Natl. Acad. Sci. U.S.A. 74:1450-1454.

9. Lazarides, E. 1980. Intermediate filaments as mechanical integrators of cellular space. Nature (London) 283:249256.

10. Lazarides, E., and D. R. Balzer, Jr. 1978. Specificity of desmin to avian and mammalian muscle cells. Cell 14: 429-438.

11. Lazarides, E., and B. L. Granger. 1978. Fluorescent localization of membrane sites in glycerinated chicken skeletal muscle fibers and the relationship of these sites to the protein composition of the $\mathrm{Z}$ disc. Proc. Natl. Acad. Sci. U.S.A. 75:3683-3687.

12. Levin, D., V. Ernst, and D. M. London. 1979. Effects of the catalytic subunit of cAMP-dependent protein kinase (Type II) from reticulocytes and bovine heart muscle on protein phosphorylation and protein synthesis in reticulocyte lysates. J. Biol. Chem. 254:7935-7941.

13. O'Connor, C. M., D. R. Balzer, Jr., and E. Lazarides. 1979. Phosphorylation of subunit proteins of intermediate filaments from chicken muscle and nonmuscle cells. Proc. Natl. Acad. Sci. U.S.A. 76:819-823.

13a. O'Connor, C. M., D. L. Gard, and E. Lazarides. 1981. Phosphorylation of intermediate filament proteins by cAMP-dependent protein kinases. Cell 23:135-143.

14. Saborio, J. L., M. Segura, M. Flores, R. Garcia, and E. Palmer. 1979. Differential expression of gizzard actin genes during chick embryogenesis. J. Biol. Chem. 254: 11119-11125.

15. Small, J. V., and A. Sobieszek. 1977. Studies on the function and composition of the 10-nm filaments of vertebrate smooth muscle. J. Cell. Sci. 23:243-268.

16. Steinert, P. M., W. W. Idler, and R. D. Goldman. 1980. Intermediate filaments of baby hamster kidney (BHK21) cells and bovine epidermal keratinocytes have similar ultrastructures and subunit domain structures. Proc. Natl. Acad. Sci. U.S.A. 77:4534-4538.

17. Suzuki, Y., L. P. Gage, and D. D. Brown. 1972. The genes for silk fibroin in Bombyx mori. J. Mol. Biol. 70: 637-649.

18. Wickner, W. 1980. Assembly of proteins into membranes. Science 210:861-868. 